\title{
A LOWER BOUND FOR THE CLASS NUMBERS OF ABELIAN ALGEBRAIC NUMBER FIELDS WITH ODD DEGREE
}

\author{
LE MAOHUA
}

(Communicated by William Adams)

\begin{abstract}
Let $\Delta_{K}, h_{K}, R_{K}$ denote the discriminant, the class number, and the regulator of the Abelian algebraic number field $K=\mathbb{Q}(\alpha)$ with degree $d$, respectively. In this note we prove that if $d>1,2 \nmid d$, and the defining polynomial of $\alpha$ has exactly $r_{1}$ real zeros and $r_{2}$ pairs of complex zeros, then $h_{K}>w \sqrt{\left|\Delta_{K}\right|} / 2^{r_{1}}(2 \pi)^{r_{2}} 33 R_{K} \log 4\left|\Delta_{K}\right|$, where $w$ is the number of roots of unity in $K$.
\end{abstract}

Let $\Delta_{K}, h_{K}, R_{K}$ denote the discriminant, the class number, and the regulator of the Abelian algebraic number field $K=\mathbb{Q}(\alpha)$ with degree $d$, respectively. In this note we prove the following result:

Theorem. If $d>1,2 \nmid d$, and the defining polynomial of $\alpha$ has exactly $r_{1}$ real zeros and $r_{2}$ pairs of complex zeros, then

$$
h_{K}>\frac{w \sqrt{\left|\Delta_{K}\right|}}{2^{r_{1}}(2 \pi)^{r_{2}} 33 R_{K} \log 4\left|\Delta_{K}\right|},
$$

where $w$ is the number of roots of unity in $K$.

Upon applying the above theorem, we can improve some known results concerning the lower bound of $h_{K}$. For instance, Barrucand, Loxton, and Williams [1] proved that if $K=\mathbb{Q}\left(D^{1 / 3}\right)$, where $D=n^{3}+m$ is not a cube, $m$ and $n$ are nonzero integers with $3 n^{2} \equiv 0(\bmod m)$, then

$$
h_{K}>\frac{0.14\left|\Delta_{K}\right|^{1 / 4}}{\log \left(\left|\Delta_{K}\right| / 3\right) \log \left(\left|\Delta_{K}\right| / 27\right)} .
$$

Notice that $r_{1}=1, r_{2}=1, w=2$, and $R_{K}<3 \log \left(\left|\Delta_{K}\right| / 3\right)$ in this case. By (1), we get a better lower bound as follows:

$$
h_{K}>\frac{\sqrt{\left|\Delta_{K}\right|}}{198 \pi \log \left(\left|\Delta_{K}\right| / 3\right) \log 4\left|\Delta_{K}\right|} .
$$

The proof of Theorem. Let $\zeta_{K}(s)$ denote Dedekind's $\zeta$-function of $K$. By [3, $\S 42]$, if $\sigma>1$, then

$$
\zeta_{K}(s)=\sum_{n=1}^{\infty} \frac{b_{n}}{n^{s}}
$$

Received by the editors August 15, 1993.

1991 Mathematics Subject Classification. Primary 11R29, 11 R20. 
where $b_{1}=1$ and $b_{n} \geq 0$ for $n>1$. Since $\zeta_{K}(2) \geq b_{1}$ and

$$
(-1)^{m} \zeta_{K}^{(m)}(2)=\sum_{n=1}^{\infty} \frac{b_{n}(\log n)^{m}}{n^{2}} \geq 0
$$

for $m>0$, we have

$$
\zeta_{K}(s)=\sum_{m=0}^{\infty} a_{m}(2-s)^{m}, \quad a_{0} \geq 1, a_{m} \geq 0, m>0,|s-2|<1 .
$$

Let $X$ be the group of Dirichlet characters associated to $K$. It is a wellknown fact that $\zeta_{K}(s)=\zeta(s) \xi_{K}(s)$, where $\zeta(s)$ is the Riemann $\zeta$-function,

$$
\xi_{K}(s)=\prod_{\substack{\chi \in X \\ \chi \neq \chi_{0}}} L(s, \chi)
$$

where $\chi_{0}$ is the trivial character and $L(s, \chi)$ is the $L$-series attached to the character $\chi$. Since $\zeta_{K}(s)$ has only simple pole at $s=1$ of residue $\xi_{K}(1)$, the function $g(s)=\zeta_{K}(s)-\xi_{K}(1) /(s-1)$ is regular. From (2), we get

$$
g(s)=\sum_{m=0}^{\infty}\left(a_{m}-\xi_{K}(1)\right)(2-s)^{m} .
$$

For any $\sigma>0$ and any $x \geq 1$, using Abel's transformation,

$$
L(s, \chi)=\sum_{1 \leq n \leq x} \frac{\chi(n)}{n^{s}}-\frac{S(x, \chi)}{x^{s}}+s \int_{x}^{\infty} \frac{S(z, \chi)}{z^{s+1}} d z,
$$

where $S(x, \chi)=\sum_{1 \leq n \leq x} \chi(n)$. Let $f_{\chi}$ denote the conductor of $\chi$. By Pólya's theorem, $|S(x, \chi)|<\sqrt{f_{\chi}} \log f_{\chi}$. By (4), we get

$$
|L(s, \chi)| \leq \sum_{1 \leq n \leq x} \frac{1}{n^{\sigma}}+\frac{2 \sqrt{f_{\chi}} \log f_{\chi}}{x^{\sigma}}<1+\frac{x^{1-\sigma}-1}{1-\sigma}+\frac{2 \sqrt{f_{\chi}} \log f_{\chi}}{x^{\sigma}} .
$$

Putting $x=\sqrt{f_{\chi}} \log f_{\chi}$. We get from (5) that

$$
|L(s, \chi)|<4 f_{\chi}^{1 / 4} \sqrt{\log f_{\chi}}<f_{\chi}^{5 / 4}, \quad \sigma \geq \frac{1}{2},
$$

since $f_{\chi} \geq 5$. Furthermore, by the conductor-discriminant formula

$$
\Delta_{K}=(-1)^{r_{2}} \prod_{\chi \in X} f_{\chi},
$$

we get from (6) that

$$
\left|\xi_{K}(s)\right|<\left|\prod_{\substack{\chi \in X \\ \chi \neq \chi_{0}}} f_{\chi}^{5 / 4}\right|=\left|\Delta_{K}\right|^{5 / 4}, \quad \sigma \geq \frac{1}{2} .
$$

Simultaneously, since $|\zeta(s)| \leq 1 /|s-1|+|s| / \sigma$ for $\sigma>0$, we have

$$
|\zeta(s)| \leq 3, \quad|s-2|=\frac{3}{2} .
$$


Therefore, by (8),

$$
\left|\zeta_{K}(s)\right|<3\left|\Delta_{K}\right|^{5 / 4}, \quad|s-2|=\frac{3}{2} .
$$

Further, by a well-known fact that $|L(1, \chi)|<\log f_{\chi}+2$, we get from (9) that

$$
|g(s)| \leq\left|\zeta_{K}(s)\right|+\left|\frac{\xi_{K}(1)}{s-1}\right|<4\left|\Delta_{K}\right|^{5 / 4}
$$

for $|s-2|=3 / 2$. Furthermore, by the maximum modulus principle, (10) holds for $|s-2| \leq 3 / 2$. Using Cauchy's theorem, we find from (3) and (10) that

$$
\left|a_{m}-\xi_{K}(1)\right|<4\left|\Delta_{K}\right|^{5 / 4}\left(\frac{2}{3}\right)^{m}, \quad m \geq 0 .
$$

Let $M$ be an integer with $M>1$. By (2) and (11), if $13 / 14<\sigma<1$, then

$$
\begin{aligned}
g(\sigma) & =\zeta_{K}(\sigma)-\frac{\xi_{K}(1)}{\sigma-1} \\
& \leq \sum_{m=0}^{M-1}\left(a_{m}-\xi_{K}(1)\right)(2-\sigma)^{m}-\sum_{m=M}^{\infty}\left|a_{m}-\xi_{K}(1)\right|(2-\sigma)^{m} \\
& <1-\xi_{K}(1) \sum_{m=0}^{M-1}(2-\sigma)^{m}-4\left|\Delta_{K}\right|^{5 / 4} \sum_{m=M}^{\infty}\left(\frac{2}{3}(2-\sigma)\right)^{m} \\
& <1-\xi_{K}(1) \frac{(2-\sigma)^{M}-1}{1-\sigma}-14\left|\Delta_{K}\right|^{5 / 4}\left(\frac{5}{7}\right)^{M} .
\end{aligned}
$$

Put

$$
M=\left[\frac{\log \left(140\left|\Delta_{K}\right|^{5 / 4}\right)}{\log (7 / 5)}\right]+1
$$

We get from (12) and (13) that

$$
\zeta_{K}(\sigma)>\frac{9}{10}-\frac{(2-\sigma)^{M}}{1-\sigma} \xi_{K}(1) .
$$

By a recent result of Chen and Wang [2], if $\chi$ is a complex character, then $L(s, \chi)$ has no zero in the range

$$
1 \geq \sigma>1-\frac{c}{\log f_{\chi}(|t|+2)}, \quad t \geq 0,
$$

where

$$
c=\max \left(0.089193, \frac{19.09712}{43.14093+12.169 / \log f_{\chi}(|t|+2)}-0.339\right) .
$$

Since $c>0.0553581$ for $f_{\chi} \geq 5$, we see that $\angle(\sigma, \chi) \neq 0$ for the range $1-1 / 18.0642 \log 2 f_{\chi} \leq \sigma<1$. Since $2 \nmid d$, all characters of $X$ are complex characters. Notice that $d \geq 3$. We get from (7) that $\left|\Delta_{K}\right| \geq f_{\chi}^{2}$ for any $\chi \in X$. Hence, $\angle(\sigma, \chi) \neq 0$ for $1-1 / 9.0321 \log 4\left|\Delta_{K}\right| \leq \sigma<1$. It implies that $\zeta_{K}(\sigma)<0$ for $1-1 / 9.0321 \log 4\left|\Delta_{K}\right| \leq \sigma<1$, and by (14), we obtain

$$
\xi_{K}(1)>\frac{9\left(1-\sigma_{0}\right)}{10\left(2-\sigma_{0}\right)^{M}},
$$


where $\sigma_{0}=1-1 / 9.0321 \log 4\left|\Delta_{K}\right|$. Since $d \geq 3$ and $\left|\Delta_{K}\right| \geq 23$, we get from (13) that

$$
\begin{aligned}
\log \left(2-\sigma_{0}\right)^{M} & =M \log \left(1+\frac{1}{9.0321 \log 4\left|\Delta_{K}\right|}\right) \\
& <\left(1+\frac{\log 140\left|\Delta_{K}\right|^{5 / 4}}{\log (7 / 5)}\right)\left(\frac{1}{9.0321 \log 4\left|\Delta_{K}\right|}\right) \\
& \leq\left(1+\frac{\log 140+\log 23^{5 / 4}}{\log (7 / 5)}\right)\left(\frac{1}{9.0321 \log 4.23}\right)<1.17
\end{aligned}
$$

and $\left(2-\sigma_{0}\right)^{M}<3.23$. Substituting it into (15),

$$
\xi_{K}(1)>\frac{1}{33 \log 4\left|\Delta_{K}\right|} \text {. }
$$

Thus, by (16) and the class number formula

$$
h_{K}=\frac{w \sqrt{\left|\Delta_{K}\right|}}{2^{r_{1}}(2 \pi)^{r_{2}} R_{K}} \xi_{K}(1)
$$

we get (1) immediately. The theorem is proved.

\section{REFERENCES}

1. P. Barrucand, J. Loxton, and H. C. Williams, Some explicit upper bounds on the class number and regulator of a cubic field with negative discriminant, Pacific J. Math. 128 (1987), 209-222.

2. J.-R. Chen and T.-Z. Wang, On the distribution of zeros of Dirichlet L-functions, Sichuan Daxue Xuebao 26 (1989), Special Issue, 145-155.

3. E. Hecke, Vorlesung über die Theorie der algebraischen Zahlen, Akademische Verlagsgesellschaft, Leipzig, 1923.

Department of Mathematics, Hunan Normal University, P.O. Box 410081, Changsha, Hunan, People's Republic of China

Current address: Department of Mathematics, Zhanjiang Teachers College, P.O. Box 524048, Zhanjiang, Guangdong, People's Republic of China 